\title{
Editorial
}

\section{Educación científica basada en pruebas}

\author{
Jordi Solbes $^{1}$ \\ Universidad de Valencia, España \\ Nidia Yaneth Torres \\ Universidad Pedagógica y Tecnológica de Colombia, Colombia
}

Editores Invitados

\section{La didáctica de las ciencias}

El sistema educativo es muy grande y, por eso, hay mucha resistencia a los cambios en la práctica educativa, debido a que los profesores están aislados en sus aulas y no forman grupos de investigación e innovación, lo que dificulta que los cambios se propaguen; y a que los profesores siguen la tradición - repiten lo que hicieron con ellos en su larga época de estudiantes-, o toman sus decisiones por ensayo y error (Gil, 1991). Frente a esto ¿es posible una educación científica basada en pruebas?

Nuestra respuesta, quizá optimista, es afirmativa. Para ello, tendríamos que dar previamente una respuesta a la pregunta: ¿qué tendríamos que "saber", "saber hacer" y "saber ser" los profesores de ciencias? La respuesta, dada por diversos autores (Gil, 1991; Porlán et al., 2010; Roth, 2007; Shulman, 1987), es el conocimiento profesional docente, que incluye una serie de saberes que permiten desarrollar una práctica docente basada en evidencias:

a. el conocimiento de la disciplina en un sentido amplio, lo que además incluye conocer la historia de las ciencias, la naturaleza de la ciencia y las metodologías que los científicos utilizan en su trabajo; las interacciones ciencia, tecnología y sociedad; y la capacidad de seleccionar y secuenciar los contenidos didácticos adecuados.

1. jordi.solbes@uv.es 
b. el conocimiento pedagógico, que incluye la gestión del aula, el modelo instruccional, la utilización de las TIC, etc.

c. el conocimiento didáctico del contenido, es decir, orientaciones y concepciones sobre la enseñanza de las ciencias, estrategias de enseñanza, conocimiento del aprendizaje e ideas de los estudiantes, métodos de evaluación, conocimiento de los currículos y materiales de aprendizaje, etc.

En estos conocimientos, la didáctica de las ciencias es el núcleo vertebrador de la formación del profesorado de ciencias. Consiste tanto en el conocimiento de las dificultades de aprendizaje del alumnado y de enseñanza del profesorado, como en el desarrollo de estrategias didácticas eficientes para superarlas. Para ello, se realizan un análisis crítico de la realidad educativa - diagnóstico- y propuestas alternativas —intervención - para transformarla (Solbes, 2009). En esto encontramos notables paralelismos con el nombre de esta revista, que se justifica en su primera editorial (Gómez, 2010). Praxis se refiere a una práctica que involucra tres procesos: transformación — de los sujetos y ambientes educativos - crítica - frente a situaciones injustas e inequitativas - y sentido — dirección a la investigación y la pedagogía-. Por su parte, el saber debe circular por los ámbitos educativos, lo que solo se logra con un énfasis importante en la investigación.

La didáctica de las ciencias apuesta por un profesorado investigador y crítico, que contribuya a comprender el mundo, a familiarizarse con los procedimientos de la investigación y a la formación del pensamiento crítico de los estudiantes, de manera que puedan tomar decisiones fundamentadas en la responsabilidad social (Torres \& Solbes, 2016). Además, en el proyecto La evaluación de la formación del profesorado de ciencias en la sociedad del conocimiento. Propuestas de mejora, se ha puesto de manifiesto que la estrategia más fructífera para mejorar la enseñanza consiste en implicar al profesorado en la investigación de los problemas de enseñanza y aprendizaje de las ciencias que les plantea su actividad docente. También se ha demostrado que hay una gran influencia de la formación intensiva y de la investigación e innovación — realizadas en didáctica de las ciencias- en la práctica docente del profesorado de ciencias (Solbes et al., 2018).

\section{Temática central}

El tema de Didáctica de las Ciencias Experimentales pretende ser un encuentro académico para conocer diferentes perspectivas pedagógicas del docente en ciencias, la problematización de la enseñanza de las ciencias en las aulas y sus implicaciones en la cultura científica de la sociedad, cuestiones que demandan una profunda reflexión. Esta sección está constituida por diez textos que abarcan diversos enfoques, modalidades y temáticas.

En el primer artículo, La configuración de la enseñanza de la biología: una inquietud por la pedagogía, Paola Andrea Roa explora la configuración de la enseñanza de la biología. Para ello, realiza una mirada genealógica al archivo -1890-2016-, que le permite decir que la biología emerge en la escuela colombiana en la primera mitad del siglo pasado. Se problematiza su integración e interdisciplinariedad con la educación ambiental y la interculturalidad.

En el segundo texto, La formación de maestros de biología: una mirada a la Universidad Pedagógica Nacional, Deysi Liseth Serrato identifica las condiciones históricas que han posibilitado la formación de maestros de biología en dicha universidad y las transformaciones 
en los planes de estudio. De la misma manera, hace una revisión de la formación disciplinar de maestros de biología, por lo cual es importante preguntarse por las relaciones entre la biología y las prácticas de formación de maestros en el escenario universitario.

En el artículo titulado Prácticas pedagógicas para la aproximación al conocimiento como científico social y natural en estudiantes de secundaria, de Alexandra Castelblanco, José Eduardo Cifuentes, Diana Patricia Pinilla y Sarasbathy Debi Pulido, se presenta una experiencia de aula para promover la aproximación al conocimiento científico natural y social en estudiantes de educación básica secundaria y media.

En el cuarto texto, Tecnologías de la información y la comunicación aplicadas a la educación en ciencias, Danny José Lorduy y Claudia Patricia Naranjo describen el uso de las TIC para desarrollar competencias tecnológicas, la creatividad y la innovación como factores asociados al aprendizaje de contenidos en ciencia, tecnología y sociedad [CTS]. Desde esta mirada, se reconocen las potencialidades del componente CTS en educación, el cual pretende la integración de los individuos con su contexto social, a través de la adquisición de los conocimientos, destrezas y actitudes necesarias para su participación en la vida social y el ejercicio de la ciudadanía en sociedades complejas y democráticas.

En el quinto artículo, Zulman Muñoz-Burbano, Jordi Solbes y Germán Ramos realizan el Análisis de la enseñanza de conceptos cuánticos en la unidad de 'Estructura atómica de la materia' en libros de texto de grado décimo en el área de química. El objetivo es indagar si en esta unidad se realiza la transposición de conceptos cuánticos básicos relacionados con la estructura atómica de la materia y cómo se hace. Concluyen que los libros analizados no utilizan los conceptos cuánticos para desarrollar dicha unidad y que se presentan omisiones, yuxtaposición de conceptos clásicos y cuánticos, e incluso errores conceptuales, sobre todo en la transposición de la dualidad de los objetos cuánticos, en las relaciones de indeterminación y en los números cuánticos.

En el artículo titulado Aprender ciencias experimentales mediante TIC en tiempos de covid-19: percepción del estudiantado, María Del Carmen Urzúa y Diana Patricia Rodríguez describen una caracterización de la enseñanza del contenido en materias teórico-prácticas como el Laboratorio de Microbiología Experimental, en el contexto de la pandemia causada por el covid-19. De esta manera, se describe cómo, a pesar de las múltiples formas y recursos tecnológicos, no se puede asegurar un cambio en la dinámica educativa debido al uso de las TIC en el área de ciencias naturales, por lo cual sugieren que estos recursos tendrían que estar asociados al aprendizaje de contenidos en el componente CTS con regularidad en las aulas.

La enseñanza de la biodiversidad se describe en el artículo de Lizeth Paola de la Cruz y Nabi del Socorro Pérez, texto que indaga sobre concepciones de los estudiantes de básica secundaria. En este se muestra cómo la mayoría de los estudiantes muestra un dominio básico de los contenidos relacionados con la biodiversidad, limitado a la variedad de organismos animales y vegetales, ya que se desestiman conceptos como diversidad genética y ecosistémica, adaptación, evolución, ecología poblacional, entre otros, que al integrarse podrían consolidar un saber sistémico y complejo.

En el octavo artículo, Método para la solución de tareas experimentales cualitativas de biología, de Dayly García, Yusimí Guerra y Julio Leyva, se aborda una problemática al realizar las prácticas de laboratorio de biología. Los autores describen como alternativa 
de enseñanza las tareas experimentales cualitativas de biología, que involucran etapas como comprensión de la tarea, confección del plan de la solución, ejecución del plan de la solución, diseño del experimento, ejecución del experimento y procesamiento de los datos, control valorativo del curso de la solución y de los resultados, contrastación teórica de los resultados y proposición de la nueva tarea.

En el noveno artículo, Dulce López Valentín propone la implementación de una secuencia didáctica basada en el modelo de aprendizaje como investigación orientada para la enseñanza del concepto de elemento químico en la educación secundaria, teniendo en cuenta las ideas previas de los estudiantes y el análisis histórico sobre este concepto. La evaluación revela que los estudiantes de los grupos experimentales presentan un mejor manejo en la definición procedimental de sustancia en el nivel macroscópico, definen mejor el concepto de elemento químico en el marco de la teoría daltoniana, distinguen mejor los conceptos de sustancia simple y elemento químico y presentan una mejor distinción del concepto microscópico de mezcla.

Y, por último, en el décimo texto, denominado Concepciones de los estudiantes de un contexto rural sobre la fotosíntesis, escrito por las profesoras Yobana Lucía Vega, Nidia Yaneth Torres y Eliana Yizeth Pedreros, se describe y se resalta la importancia de la enseñanza de la fotosíntesis como tema integrado de las ciencias naturales, que cobra interés porque permite abordar la diferencia entre conceptos como nutrición, respiración y rutas metabólicas. El documento narra las concepciones que tienen estudiantes del contexto rural sobre su enseñanza y constituye un aporte que reconoce el lenguaje científico como un sistema de recursos para construir significados y la necesidad de centrarse en las estrategias retóricas que adoptan los estudiantes en la comprensión de conceptos.

\section{Investigación y pedagogía}

En esta sección, hay cinco artículos que se enmarcan en el segundo componente del conocimiento profesional docente: el conocimiento pedagógico. En el primer texto, denominado Instituciones normalizadoras para anormales, de Elsa Georgina Aponte, se describen interesantes investigaciones que permiten analizar las instituciones como parte de la constitución de sujetos anormales. La investigación está ubicada entre los años 40 y 70 del siglo XX.

En el artículo titulado Aproximaciones sobre los discursos y prácticas de la Escuela Activa en Colombia, de Luz Andrea Gutiérrez y Liliana Andrea Mariño, se presenta una construcción teórico-reflexiva como estado del arte de la Escuela Activa en Colombia, el cual hace parte del proyecto Escuela Activa.

De la misma manera, en el tercer artículo, Iván Darío Cruz, Lizeth Ximena Castro y Manuel Alejandro Ojeda evidencian la necesidad de configurar una didáctica de la filosofía y sus conceptualizaciones desde el Conocimiento didáctico del contenido y enseñanza de la filosofía. Su intención es describir las potencialidades y limitaciones sobre la enseñanza del saber filosófico.

En el cuarto artículo, Desempeño pedagógico docente y aprendizaje de los estudiantes universitarios en la carrera de Educación, de Llésica Soria, Wilmer Ortega y Arnulfo Ortega, se muestra la relación entre el desempeño docente y el aprendizaje de estudiantes de las 
carreras profesionales de Educación Inicial y Educación Primaria, de la Universidad Nacional de Ucayali-Pucallpa.

Finalmente, desde el texto denominado El pensamiento decolonial como una alternativa al 'racismo inverso' en el fútbol, de Otávio Nogueira, João Alberto Steffen y Gilberto Ferreira da Silva, se aborda el tema del "racismo inverso" en el fútbol, considerado como otro mecanismo de subyugación de la élite blanca, y las críticas a este prejuicio.

\section{Conclusiones y perspectivas}

Los artículos presentados examinan propuestas alternativas de enseñanza en diversos conceptos delas ciencias, que han sido evaluadas y que, por lo tanto, constituyen conocimiento basado en pruebas. Un aspecto que se destacó en el número es la problematización de prácticas de laboratorio en diversos escenarios educativos y el análisis de libros de textos, para diagnosticar la enseñanza realizada, ya que no se puede elaborar una propuesta efectiva si previamente no se conoce a profundidad la situación problemática de partida.

Dentro de las perspectivas se destaca la necesidad de desarrollar una mayor comprensión sobre las estrategias para apoyar el desarrollo profesional de los docentes de ciencias mediante las innovaciones e investigaciones en la enseñanza de las ciencias, para disponer de conocimientos profesionales docentes fundamentados en evidencias.

\section{Referencias}

Gil, D. (1991). ¿Qué han de saber y saber hacer los profesores de ciencias? Enseñanza de las Ciencias, 9(1), 69-77.

Gómez, L. (2010). Editorial. Praxis \& Saber, 1(1), 13-17.

Porlán, R., Martín, R., Ribero, A., Harres, J., Azcárate, P., \& Pizzato, M. (2010). El cambio del profesorado de ciencias I: Marco teórico y formativo. Enseñanza de las Ciencias, 28(1), $31-46$.

Roth, K. (2007). Science Teachers as Researchers. En S. Abell, \& N. Lederman (Eds.), Handbook of Research on Science Education (pp. 1205-1259). Routledge.

Shulman, L. (1987). Knowledge and teaching: Foundations of the new reform. Harvard Educational Review, 57(1), 1-22. https://doi.org/10.17763/haer.57.1.j463w79r56455411

Solbes, J. (2009). Dificultades de aprendizaje y cambio conceptual, procedimental y axiológico (I): resumen del camino avanzado. Revista Eureka sobre Enseñanza y Divulgación de las Ciencias, 6(1), 2-20.

Solbes, J., Fernández, J., Dguez-Sales, M., Cantó, J., \& Guisasola, J. (2018). Influencia de la formación y la investigación didáctica del profesorado de ciencias sobre su práctica docente. Enseñanza de las Ciencias, 36(1), 25-44 https://doi.org/10.5565/rev/ ensciencias. 2355

Torres, N., \& Solbes, J. (2016). Contribuciones de una intervención didáctica usando cuestiones sociocientíficas para desarrollar el pensamiento crítico. Enseñanza de las ciencias, 34(2), 43-65. https://doi.org/10.5565/rev/ensciencias. 1638 